\title{
BLIND DEBLURRING OF FOREGROUND-BACKGROUND IMAGES
}

\author{
Mariana S. C. Almeida and Luís B. Almeida \\ Instituto de Telecomunicações and Instituto Superior Técnico, Lisbon, Portugal \\ (mariana.almeida, lbalmeida)@1x.it.pt
}

\begin{abstract}
This paper presents a method for deblurring an image consisting of two layers (a foreground layer and a background layer) which have suffered different, unknown blurs. This is a situation of practical interest. For example, it is common to find images in which we have a foreground object (e.g. a car) which has motion blur while the background is sharp (or vice-versa), or in which a foreground object and the background have different out-of-focus blurs.

We develop a model for this foreground + background degradation, and extend a previously introduced blind deblurring method to deal with this situation. As in the original blind deblurring method, the method presented here does not impose any strong constraints on the blurring filters. The method is almost completely blind, requiring, form the user, just a coarse indication of which are the foreground and background areas of the image.

The method has been tested with synthetic degradations and with real-life photos. We present some of the results. In all the experiments, the method was able to reasonably recover, from single degraded images: the complete deblurred image, the deblurred foreground and background images, and a mask providing the segmentation between foreground and background.
\end{abstract}

Index Terms - Blind image deconvolution, Space-variant blur, Image enhancement, Object segmentation.

\section{INTRODUCTION}

Image deblurring is an inverse problem whose aim is to recover images that have suffered blurring degradations. The blurring degradations can be shift-invariant (modeled by a convolution) or shiftvariant (approximated by local convolutions). The present manuscript focuses on a special class of shift-variant degradations, in which two different regions of the image (that we call foreground and background, respectively) have different blurs. This is a situation often encountered in photography, when the scene is composed of an object that is closer to the camera, and a farther background. In this case, two typical scenarios are: (1) the closer object has motion blur and the background scene is static and in focus (or vice-versa); (2) the closer object and the farther background are both stationary, but have different focus blurs. These are two scenarios on which we make experimental tests in this paper, but it should be clear that the method to be presented is much more general, allowing the recovery from wide classes of degradations both in the foreground and in the background. In the context of this paper, we shall call the images composed of foreground and background "two-layer images", and the corresponding blurs "two-layer blurs", while the ordinary images, with shift-invariant blurs, shall be called "single-layer".

This work was partially supported by the PhD fellowship SFRH/BD/23919/2005 from FCT, Portugal.
In single-layer blind image deconvolution (BID), both the image and the blur operator are unknown. This is an ill-posed problem, since there is an infinite number of pairs (original image + blurring filter) that could have led to the observed degraded image. Additionally, the blurring operator of BID typically is very ill-conditioned, making the estimate of the deblurred image very sensitive to noise and to mismatches between the estimated blurring filter and the true one. In shift-variant blind deblurring, the number of parameters to be estimated is even larger than in shift-invariant BID. In spite of the fact that the problem is rather challenging, a few shift-variant deblurring methods have been developed $[1,2,3,4,5,6,7,8]$. In $[1,2,4,5,6]$, the problem's difficulty is reduced by using multiple degraded images of the same scene. Results presented in [3] assume a highly restricted blur model (only out-of-focus), which is only tested in a simple synthetic image. $[5,6,7]$ address problems that are similar to those of the present work. However, $[5,6]$ use multi-frame scenarios. The method of [7] is restricted to out-offocus degradations and does not correctly treat the regions' boundaries. Furthermore, the methods presented in $[5,6,7]$ only address parameterized blurs, while the one that we present here handles more generic, non-parametric blurs. The method in [8] is restricted to motion blurs and does not estimates a sharp image.

The approach that we propose in this paper evolved from a previously developed single-layer blind deblurring method $[9,10]$. That method can satisfactorily deblur a wide range of degradations, without imposing any strong restrictions on the blurring filter. The flexibility of that method carries on to the method that we propose here, which can deal with rather unrestricted blurs in both in the foreground and the background layers. The method is quasi-blind, requiring only a crude initial guess of the foreground and background zones, for region segmentation. The method's performance was satisfactory when tested with synthetic degradations of natural images and with an actual photograph.

The structure of this paper is as follows: The single-layer deblurring method is briefly reviewed in Section 2. The two-layer method is then presented in Section 3. Section 4 reports experimental results.

\section{SINGLE-LAYER METHOD}

We consider the blurring model

$$
y=h * x+n,
$$

in which $x$ is the original image, $h$ is the PSF (point spread function) of the blurring filter and $n$ is additive noise. The symbol $*$ denotes mathematical convolution.

The deblurring method is based on two basic observations:

- In natural images, edges are sparse.

- In general, edges of a blurred image are less sparse than those of a sharp image, because they occupy a wider area (here, 


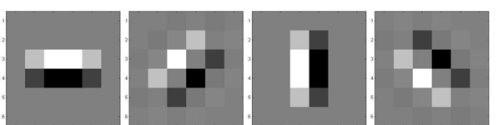

Fig. 1. The set of edge detection filters, in the four orientations that were used.

"edges" refers to continuously-valued variables that are related to the speed of spatial variation of the image's intensity).

These two observations lead to the method's central idea: using an objective function which favors images with sparse edges. The objective function that is used is of the form

$$
C=\|y-\hat{y}\|_{2}^{2}+\lambda_{x} R_{f}(x),
$$

in which $y$ is the observed blurred image, $\hat{y}$ is the estimate of the blurred image, obtained through $\hat{y}=h * x$, where $x$ is the estimated original image and $h$ is the estimated blurring filter. $R_{f}(x)$ is a regularizer which favors images with sparse edges. It uses an edge detector based on several directional edge-detecting filters (see Fig. 1). The output of the edge detector is given by

$$
f(x)=\sqrt{\sum_{\theta \in \Theta} g_{\theta}^{2}},
$$

where $g_{\theta}$ is the output of the edge detection filter with orientation $\theta$ and $\Theta$ is the set of orientations under consideration. The regularizer $R_{f}($.$) operates on the edge intensity image f$ from Eq. (3):

$$
R_{f}(x)=R(f(x))=\sum_{i}\left(f_{i}+\epsilon\right)^{q},
$$

where $i$ is an index running through all pixels, $q$ controls the prior's sparsity and $\epsilon$ is a small parameter which yields finite lateral derivatives at $f=0$ (with $0<q<1$ ), making the corresponding prior closer to actual distributions and also making the optimization easier.

Direct minimization of the objective function in (2), by itself, doesn't generally lead to good deblurring results. The deblurring method uses a guiding procedure which essentially consists of starting with a large value of $\lambda$ (heavy regularization) and progressively decreasing it as the estimate of the blurring filter becomes better. This guiding procedure allows the method to be able to deblur a wide range of natural and artificial images with a wide range of blurs, without imposing any strict constraints on the blur PSF. References $[9,10]$ give more details, as well as experimental results.

\section{TWO-LAYER METHOD}

We now designate by $x_{1}$ the background image, by $x_{2}$ the foreground image, and by $o$ an "opacity" mask, which describes the opacity of the foreground layer: If the foreground only contains opaque objects, $o$ is a binary mask having ones in the pixels corresponding to the foreground objects and zeros in those corresponding to the background scene. If the foreground contains semi-transparent objects, then $o$ is supposed to also contain that transparency information.

We assume that a sharp image would be formed according to

$$
x_{r}=x_{1} \cdot(1-o)+x_{2} \cdot o .
$$

The operator - denotes the Hadamard product. We model the blurred image by

$$
y=\left(h_{1} * x_{1}\right) \cdot(1-\hat{o})+\left(h_{2} * x_{2}\right) \cdot \hat{o}+n,
$$
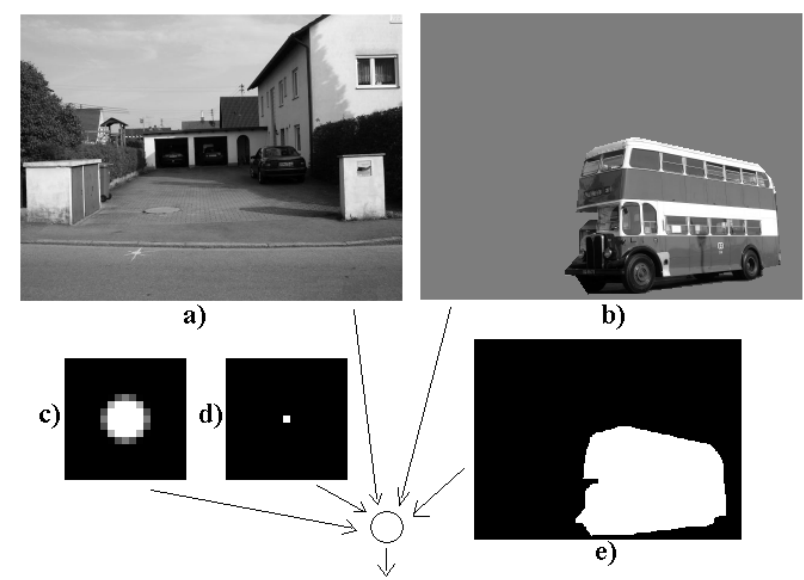

b)
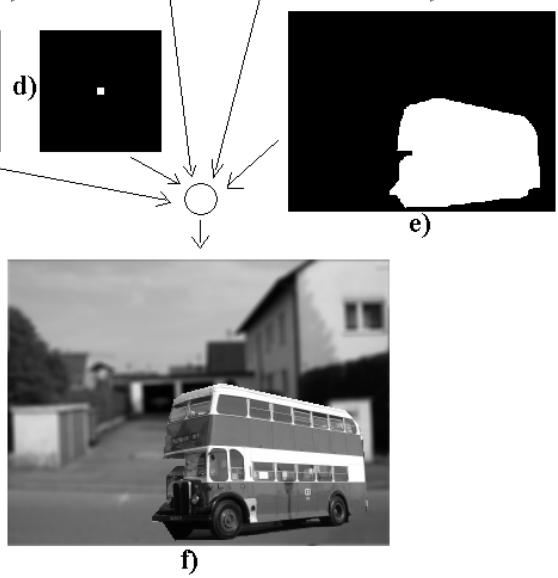

e)
Fig. 2. First synthetic degradation. a) Background image. b) Foreground object. c) Background blurring filter. d) Foreground blurring filter. e) Region segmentation. f) Blurred image.

in which $h_{1}$ is the PSF of the blurring operator that has degraded the background image, and $h_{2}$ is the PSF of the blurring operator that has degraded the foreground image. The operator $*$ denotes the mathematical operation of convolution. $n$ represents additive noise, and $\hat{o}$ is the blurred opacity mask, given by

$$
\hat{o}=h_{2} * o
$$

The estimation procedure is an extension of the single-layer one briefly presented in Section 2. All unknowns $\left(x_{1}, x_{2}, h_{1}, h_{2}\right.$ and $o$ ) are estimated by minimizing the cost function

$$
C=\|y-\hat{y}\|_{2}^{2}+\lambda_{x}\left(R_{f}\left(x_{r}\right)+R_{f}\left(x_{1}\right)+R_{f}\left(x_{2}\right)\right)+\lambda_{o} R_{f}(o),
$$

in which $\hat{y}$ is the estimate of the blurred image,

$$
\hat{y}=\left(h_{1} * x_{1}\right) \cdot(1-\hat{o})+\left(h_{2} * x_{2}\right) \cdot \hat{o},
$$

and $x_{r}$ is the estimated sharp image, which is constructed as in (5). $\lambda_{x}$ is a regularizing parameter that controls the regularization applied to the estimated images (complete image, background and foreground), and $\lambda_{o}$ is another regularizing parameter, controlling the regularization applied to the opacity mask $o$.

Like the single-layer method, the two-layer method only restricts the blurring filters to have a limited support. Among the infinite number of solutions, a suitable one is reached by starting with a large value for the regularizing parameters $\left(\lambda_{x}\right.$ and $\left.\lambda_{o}\right)$ and progressively reducing them. Having two regularizing parameters $\left(\lambda_{x}\right.$ and $\left.\lambda_{o}\right)$, it is convenient to fix a relationship between them. A good value for the ratio $\left(\lambda_{o} / \lambda_{x}\right)$ was empirically found to be 0.1 .

For efficiency reasons, the images $\left(x_{1}, x_{2}\right)$, the filters $\left(h_{1}, h_{2}\right)$ and the segmentation $(o)$ are not simultaneously optimized. Table 1 outlines the method, for which a decreasing sequence of $\lambda_{x}$ (and, 
consequently, of $\lambda_{o}$ ) values and a non-increasing sequence of $q$ values are assumed to have been previously chosen ( $q$ controls the regularizer's sparsity, as we'll see ahead).

\section{Initialization}

$1-$ Set $h_{1}$ and $h_{2}$ to the identity operator

$2-$ Set $x_{1}$ and $x_{2}$ equal to $y$.

3 - Initialize $o$ (as detailed in Section 3.1)

$4-\operatorname{Set} \lambda_{x}, \lambda_{o}$ and $q$ to the initial values of their sequences.

Optimization loop:

5 - Find new estimates for $x_{1}$ and $x_{2}$ :

$\left(x_{1}, x_{2}\right)=\operatorname{argmin}_{x_{1}, x_{2}} C\left(x_{1}, x_{2}, h_{1}, h_{2}, o\right)$

6 - Find a new $o$ estimate: $o=\operatorname{argmin}_{o} C\left(x_{1}, x_{2}, h_{1}, h_{2}, o\right)$

7 - Find new estimates for $h_{1}$ and $h_{2}$ :

$\left(h_{1}, h_{2}\right)=\operatorname{argmin}_{h_{1}, h_{2}} C\left(x_{1}, x_{2}, h_{1}, h_{2}, o\right)$

8 - Set $\lambda_{x}, \lambda_{o}$ and $q$ to the next values in sequence.

9 - If $\lambda_{x} \geq \lambda_{x_{\text {min }}}$ go back to 5; otherwise stop.

Table 1. Method for deblurring a single image composed by two differently blurred areas.

During early iterations of the method, $\lambda_{x}$ is large and only the main edges of the estimated image will survive, but they will be sharp $[9,10]$. The sharp edges of the over-regularized images lead the estimates of the blurring operators to improve, and this, in iteration, allows smaller and fainter features to be progressively estimated, at the same time that the estimates of the blurring operators also improve. The gradual reduction of $\lambda_{x}$ and $\lambda_{o}$ results in a guiding technique which progressively considers smaller and fainter features.

\subsection{Prior information and preprocessing}

The method needs to be initialized with a small amount of prior information, which is easily provided by a human. It consists of the indication of two compact regions in the image: one that is known to correspond to the background area, and another one that is known to correspond to the foreground area. Once these regions have been assigned, a preprocessing phase applies a segmentation algorithm [11] to find an initial estimate $\left(o_{0}\right)$ for the $o$ mask (step 3 of Table 1). The method of [11] was implemented using horizontal and vertical image differences as features, with probability density functions that were estimated from the statistics of the assigned areas.

\section{EXPERIMENTS}

The proposed method was tested with synthetic and real-life degradations. The edge detector was implemented using the filters shown in Fig. 1, which were obtained from the basic filter

$$
F_{\theta=0}=\left[\begin{array}{cccc}
1 & 2 & 2 & 1 \\
-1 & -2 & -2 & -1,
\end{array}\right] / 12
$$

by applying rotations by multiples of $45^{\circ}$. For all the experiments, we have used the same settings as in [9]: the images were normalized so that their intensity values would cover the interval $[-0.5,0.5] ; \epsilon$ was set to 0.002 ; the sequence of values for parameter $q$ was: 0.8 , $0.8,0.6,0.6,0.6,0.6,0.4, \ldots, 0.4$; the sequence for $\lambda_{x}$ was geometric: $\lambda_{x, n+1}=\lambda_{x, n} / r$ with $\lambda_{x, 0}=2$, and with values of $r$ that are given ahead. The sequence of values of $\lambda_{x}$ was manually truncated at a value which led to the best compromise between image detail and noise or artifacts. We have manually chosen $\lambda_{o} / \lambda_{x}=0.1$, as said above. The supports of the blurring filter estimates $\left(h_{1}\right.$ and $\left.h_{2}\right)$ were

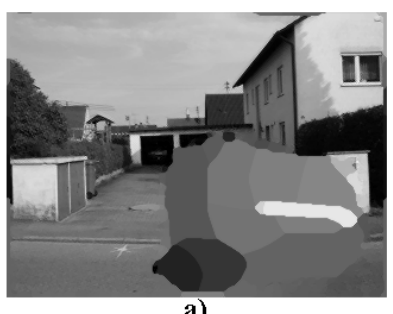

a)
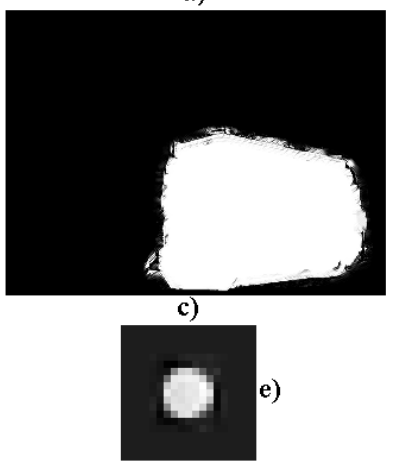

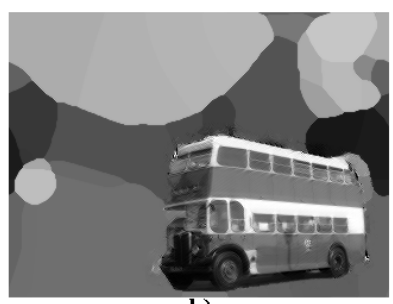

b)

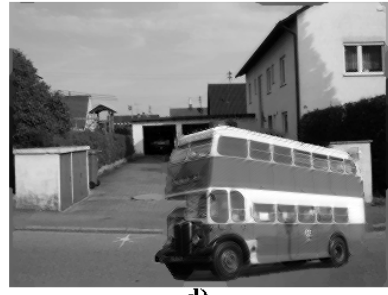

d)

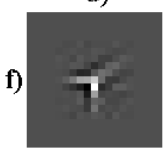

Fig. 3. Results of the first synthetic experiment. a) Estimated background image, b) estimated foreground image, c) segmentation, d) estimated sharp scene, e) estimated background blur, f) estimated foreground blur.

limited to squares of size $11 \times 11$ pixels each, and we used a safety margin of width 3 around these supports (see $[9,10]$ for details). All minimizations (steps 5, 6 and 7 of Table 1) were performed by gradient descent with adaptive step sizes.

In the first set of experiments, the method was tested with synthetic degradations. The sharp scene was constructed from two natural sharp images, corresponding to the background (Fig. 2-a) and foreground (Fig. 2-b). This sharp scene had $240 \times 320$ pixels and was subject to three different synthetic degradations: in the first degradation (Fig. 2-f) the foreground image was sharp and the background one had an out-of-focus blur; in the second degradation (Fig. 4-a) the foreground image had motion blur and the background was sharp; the third degradation (Fig. 5-a) combined the previous two degradations: The foreground image had motion blur and the background one had an out-of-focus blur.

For these experiments, we have set $r=2$ and the sequence of $\lambda$ values was stopped at $\lambda_{x, 24}=1.19 \times 10^{-7}$. Fig. 6-a shows the manually chosen foreground and background regions that were used for this set of experiments. white corresponds to the manually chosen foreground region, black to the manually chosen background region, and gray to neither. The estimates obtained for the first degradation are shown in Fig. 3. For the other two degradations we only show the sharp scene and the blurring filter estimates, due to lack of space (Figs. 4-b,c,d and 5-b,c,d). All these experiments led to sharper reconstructed images, in which more details were visible than in the corresponding blurred images. The estimated blurring filters and segmentation were also close to the true ones.

Another experiment was made with a real-life degradation corresponding to an actual photo obtained from the web (Fig. 7-a). The manually selected segments used for this experiment are shown in Fig. 6-b. We have set $r=1.5$ and the iteration was stopped at $\lambda_{x, 23}=1.78 \times 10^{-4}$. As we see from Fig. 7-b, the estimated scene was sharper than the original. In judging these results, one should take into account that photos normally involve some non- 


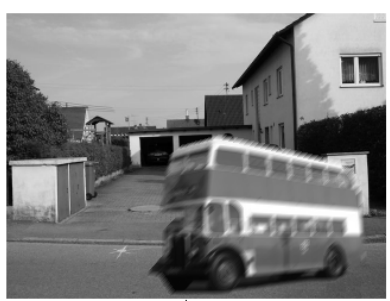

a)

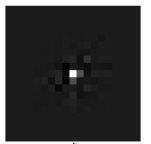

c)

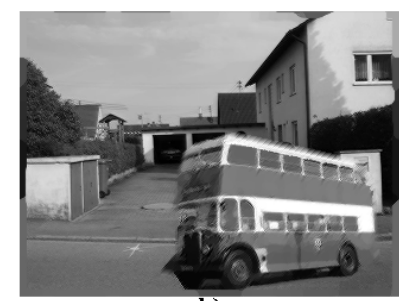

b)

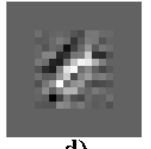

d)
Fig. 4. Second synthetic experiment. a) Blurred image. b) Estimated sharp scene. c) Estimated background blur. d) Estimated foreground blur.

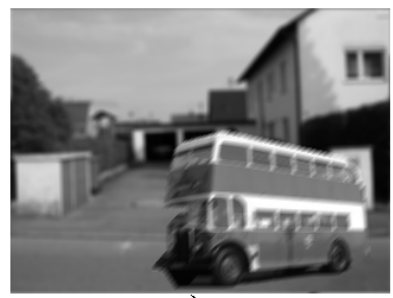

a)

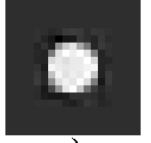

c)

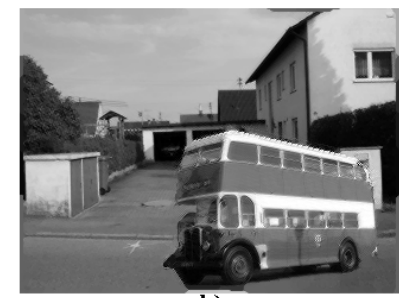

b)

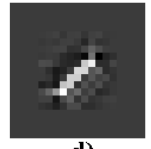

d)
Fig. 5. Third synthetic experiment. a) Blurred image. b) Estimated sharp scene. c) Estimated foreground blur. d) Estimated background blur.

linear processing, and thus do not exactly conform to our blurring model.

\section{CONCLUSIONS AND FUTURE WORK}

We have presented a method for deblurring shift-variant image degradations which often occur in photography. This kind of degradations occurs when the photographed scene has a foreground object and a farther background. The approach presented in this paper is an extension of the shift-invariant BID method [9, 10]. As happens with that method, the one proposed here does not impose any strong restrictions on the blurring filter. The method's performance was tested on synthetic and real-life degradations.

In the future, we plan to extend the presented method in order to address more than two blurred regions. We also plan to extend the originating BID method $[9,10]$ to address other shift-variant degradations of practical interest.

\section{REFERENCES}

[1] M. Blume, D. Zikic, W. Wein, and N. Navab, "A new and general method for blind shift-variant deconvolution of biomedical images," in $\operatorname{MICCAI}(1), 2007$, pp. 743-750.

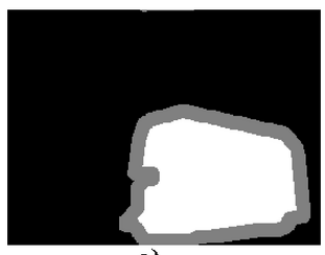

a)

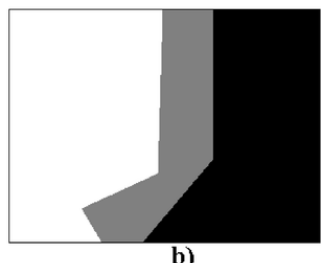

Fig. 6. Manual supervision. a) Synthetic experiments. b) Photo. White: foreground. Black: Background. Gray: Unspecified.

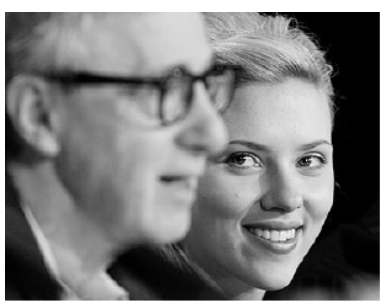

a)

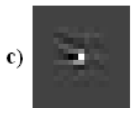

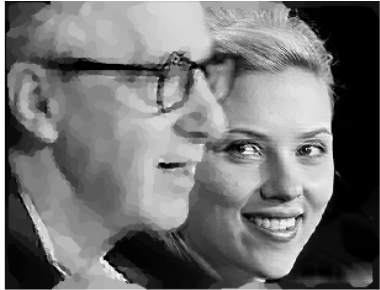

b)

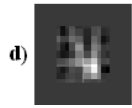

Fig. 7. Deblurring a real photo. a) Blurred photo. b) Estimated sharp scene. c) Estimated background blur. d) Estimated foreground blur.

[2] M. Sorel and J. Flusser, "Space-variant restoration of images degraded by camera motion blur," IEEE Transactions on Image Processing, vol. 17, no. 2, pp. 105-116, February 2008.

[3] Y-L. You and M. Kaveh, "Blind image restoration by anisotropic regularization," IEEE Trans. Image Process, vol. 8, no. 3, pp. 396-407, March 1999.

[4] M. Welk, D. Theis, and J. Weickert, "Variational deblurring of images with uncertain and spatially variant blurs," in DAGMSymposium, 2005, pp. 485-492.

[5] A. Kubota and K. Aizawa, "Reconstructing arbitrarily focused images from two differently focused images using linear filters," IEEE Trans. Image Process, vol. 14, no. 11, pp. 18481859, Nov 2005.

[6] L. Bar, B. Berkels, M. Rumpf, and G. Sapiro, "A variational framework for simultaneous motion estimation and restoration of motion-blurred video," in IEEE 11th International Conference on Computer Vision, 2007.

[7] J. Shin, S. Hwang, K. Kim, J. Kang, S. Lee, and J. K. Paik, "Blur identification and image restoration based on evolutionary multiple object segmentation for digital auto-focusing," in IWCIA, 2004, pp. 656-668.

[8] Shengyang Dai and Ying Wu, "Estimating space-variant motion blur without deblurring.," in ICIP, 2008, pp. 661-664.

[9] M. S.C. Almeida and L. B. Almeida, "Blind deblurring of natural images," in ICASSP, Las Vegas, March 2008.

[10] M. S.C. Almeida and L. B. Almeida, "Blind and semi-blind deblurring of natural images," IEEE Trans. Image Process, (submitted).

[11] M. Figueiredo, D. Cheng, and V. Murino, "Clustering under prior knowledge with application to image segmentation," in NIPS, 2006. 